\title{
Optimal Control for Haptic Rendering: Fast Energy Dissipation and Minimum Overshoot
}

\author{
Thomas Hulin ${ }^{1}$, Ricardo González Camarero ${ }^{2}$, Alin Albu-Schäffer ${ }^{1}$
}

\begin{abstract}
Controlling haptic devices in an optimal way is crucial to achieve both, best performance and most realistic haptic feedback. The present article investigates control design of a single degree of freedom haptic device that is interacting with a human operator and rendering a virtual wall affected by time delay. To this end, it suggests different optimization criteria based on the step response of the haptic system. These criteria cover fundamental requirements for efficiently using haptic devices, particularly fast settling and minimum overshoot. For each criterion an optimal path and point inside the stable region of the virtual wall parameters is derived. These optima depend mainly on the system mass, sampling time and time delay. This approach is supported by experiments on two devices, a Falcon haptic device and a DLR/KUKA Light-Weight Robot arm.
\end{abstract}

\section{INTRODUCTION}

The purpose of force-feedback haptic devices is to display forces from virtual or real environments to human operators. Stability is a fundamental requirement for such devices, as violating stability may result in uncontrollable oscillations that make any reasonable interaction impossible, or can even harm the human operator. Thus, lots of research in this field tackles the question of how to obtain stable control behavior for haptic interaction.

One strategy uses passivity to obtain stable systems. Passivity has the appealing advantage that it guarantees stability also if the environment is unknown. The most famous work following this strategy in the Laplace domain is written by Colgate and Schenkel [1]. They derived a passivity condition for virtual environments such that energy can never be extracted from the haptic system. Although passivity provides a comfortable means for obtaining stability, it has the disadvantage of being conservative in terms of stability [1], [2]. This means that there are parameter values that violate the passivity condition but result in a stable system behavior, possibly with even higher performance than achievable by passivity.

Another well studied control approach is the time-domain passivity controller introduced by Hannaford and Ryu [3], which observes the energy generated by the haptic device. As soon as energy is generated, a variable damper tries to dissipate this amount of energy before the system becomes unstable. This approach is less conservative compared to passivity criteria in the Laplace domain. It was improved with respect to various aspects, e.g. in [4], [5], [6]. Yet, the

\footnotetext{
${ }^{1}$ T. Hulin and A. Albu-Schäffer are with the Institute of Robotics and Mechatronics, DLR (German Aerospace Center), D-82234 Wessling, Germany. \{Thomas.Hulin, Alin.Albu-Schaeffer\}edlr.de

${ }^{2} \mathrm{R}$. González Camarero is with the Applied Mechanics Department, CEIT and TECNUN, University of Navarra, San Sebastián, E-20018, Spain.
}

practical use of this approach reveals some problems, such as imprecise energy observation and possibly delayed energy dissipation, causing the haptic system to become active.

The theoretical stability region for haptic walls represented by a virtual spring-damper system was first determined numerically by Salcudean and Vlaar [7]. They considered their haptic device as a simple mass actuated by a one sample-step delayed force. For this system, they determined the stability boundary inside a normalized parameter plane.

A more recent theoretical approach [8] analyzed stability for haptic rendering comprising time-delay and a more generic haptic device modeled as mass-damper system. In [9] this approach was enhanced by including a linear model of the human arm. That work derives stability boundaries for the parameters of the virtual wall and analyzes the influence of the human arm parameters. Although it clarifies for which wall parameters the system is stable, it becomes not clear what the optimal parameters for a specific task are. Before investigating this issue, we postulate four common objectives for an optimal haptic system behavior, which are congruent with those of many fields other than haptics:

1) high stiffness range of the wall, such that various environments can be rendered realistically, and that the steady-state position error can be minimized,

2) fast settling, such that oscillations decline quickly,

3) minimum overshoot, such that the maximal wall penetration is close to the steady-state one, and

4) robustness against parameter uncertainties, such that the human operator influences the optimal behavior only minimally.

In practice, parameter tuning for haptic interaction is often performed directly on a haptic system, following the trial and error approach. One reason for not using tuning rules is that there are no such general rules for optimally parameterizing the controller of a haptic device.

The present article finds optimal points based on the step response of impedance-type haptic systems. At first, Sect. II defines the considered haptic system. Then, Sect. III summarizes previous stability analysis on which the introduced approach is based. Sect. IV introduces optimization criteria and presents the theoretical optimal curves and point. These findings are supported by experiments in Sect. V. Sect. VI summarizes the results and draws important conclusions for the optimal control of haptic rendering. 


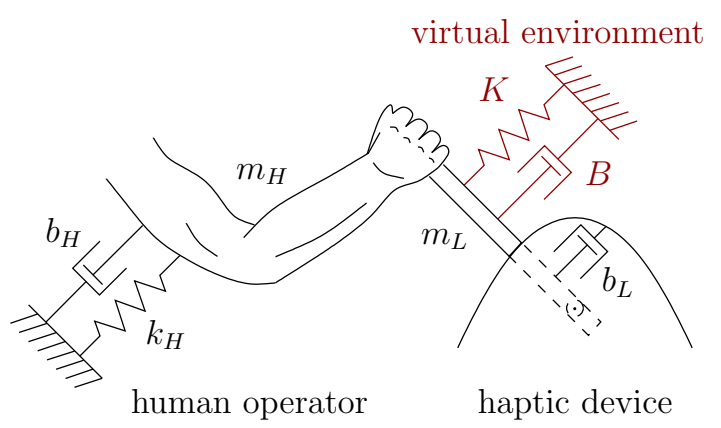

Fig. 1. Linear model of a human arm interacting with a single degree of freedom haptic device, which is rendering a discrete-time spring-damper system, [9].

\section{SYSTEM DESCRIPTION}

The control design in this article is based on the stability analysis of [9]. Thus, the present article considers the same haptic system composed of a human arm holding a haptic device (see Fig. 1). The device is colliding against a virtual wall, which is represented by a discrete-time spring-damper system (discrete-time PD-controller), with stiffness $K$, damping $B$, and constant sampling rate $1 / T$. The unilateral constraint of virtual walls is not considered.

The haptic device is modeled by a single degree of freedom mass $m_{L}$, which is damped by a viscous damper $b_{L}$. Nonlinear effects like static friction or quantization and saturation of sensors and actuators are not taken into account. Also the structural compliance the actuator dynamics of the device are neglected, such that a force will be assumed to be applied constantly over one sampling period $T$.

Each real haptic system is affected by time delay, originating from different sources, such as communication or force computation. The overall time delay $t_{d}$ is considered as sum of all delays in the haptic system, and is assumed being constant and positive $t_{d} \geq 0$. It has a transfer function of $e^{-t_{d} s}$, or in the discrete-time domain $z^{-d}$, where $d$ is the delay factor given by $d=t_{d} / T$.

The human arm is modeled by a single degree of freedom mass-spring-damper system with mass $m_{H}$, stiffness $k_{H}$ and viscous damping $b_{H}$. Although it is an approximation, this linear model of the real human has been applied in many theoretical studies [10]. It is further assumed that the human operator holds the haptic device in such a way that the human arm mass $m_{H}$ is directly coupled to the device inertia $m_{L}$. Thus, the physical parameters of the haptic device and the human can be combined to

$$
\begin{array}{rrr}
m= & m_{L}+m_{H} \\
b & = & b_{L}+b_{H} \\
k & = & k_{H},
\end{array}
$$

where $m, b$ and $k$ are the effective physical mass, damping and stiffness respectively.

Under these assumptions the control loop shown in Fig. 2 results. It contains continuous-time (physical stiffness, damping and mass) and discrete-time (virtual environment) elements. The input $F$ is an external force, comprising the

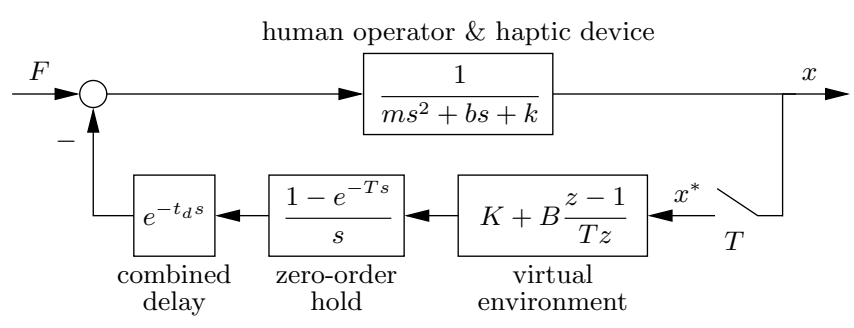

Fig. 2. Control loop of a haptic system employed for stability and passivity analysis, [9].

intended force of the human operator and possible collisions of the haptic device with the real environment. This force is assumed being constant during a sampling period $T$.

The possible range for the physical system parameters is wide. They depend not only on the haptic device, but also on the direction of movement and on the current arm and device configuration. Thus, for the calculations in this article only limits for the parameter ranges of the fractions $k / m$ and $b / m$ will be assumed, and not specific parameter values of the human and the device. These limits were derived in [9]. To account for inaccuracies in determining the parameters of the human and the haptic device, a range extended by roughly $50 \%$ will be assumed in the remainder of this article, i.e.

$$
\begin{aligned}
& 0 \leq k / m<1000 \mathrm{~s}^{-2} \\
& 0 \leq b / m<20 \mathrm{~s}^{-1} .
\end{aligned}
$$

Thus, the majority of realistic values of human arm and haptic device parameters should be covered by these parameter ranges.

\section{Summary OF ThE Stability AnAlysis}

The present work is based on a previous stability analysis of the considered system [9]. This analysis yielded stable regions and their boundaries for the discrete-time parameters of the virtual environment. This section summarizes the approach and its main results.

The discrete- and continuous-time elements of the system in Fig. 2 must be transformed into a common time domain before analyzing stability becomes possible. For the continuous-time element an exact discrete-time equivalent can be calculated, which has at the sampling instances the same behavior as the original system. This equivalent makes possible to determine the closed-loop discrete-time transfer function from force to position $G_{x}(z)=x(z) / F(z)$.

It turns out that this transfer function can be significantly simplified by certain normalization rules for the system parameters (see Table I). By applying these rules, the two parameters $m$ and $T$ drop out of the characteristic equation of the transfer function. The resulting normalized characteristic equation only depends on the five remaining dimensionless parameters. The dependency on $m$ and $T$ is implicit.

A stability check of the investigated system can now be performed easily by computing the zeros of the normalized characteristic equation. The system is stable if all zeros are located inside the unit circle in the plane of the complex variable $z$. Analytical solutions for stability could be determined 
TABLE I

NORMALIZATION RULES AND THE RESULTING DIMENSIONLESS PARAMETERS, [9].

\begin{tabular}{lcl}
\hline parameter & variable & dimensionless variable \\
\hline sampling period & $T$ & - \\
mass & $m$ & - \\
virtual stiffness & $K$ & $\alpha:=K T^{2} / m$ \\
virtual damping & $B$ & $\beta:=B T / m$ \\
physical stiffness & $k$ & $\gamma:=k T^{2} / m$ \\
physical damping & $b$ & $\delta:=b T / m$ \\
delay & $t_{d}$ & $d:=t_{d} / T$ \\
\hline
\end{tabular}

by applying the Jury stability criterion [11]. Yet, for delays larger than zero the resulting expressions quickly become very complex. Thus, in [9] an iterative method is applied to numerically determine points on the stability boundaries.

Fig. 3 shows the resulting boundaries for fixed delays $d=0$ (left) and $d=1$ (right) in the parameter plane of the virtual environment. It also shows the influence of the normalized physical damping $\gamma$ and stiffness $\delta$ inside their possible parameter ranges. These ranges for the normalized parameters can be determined by combining the limits of the parameter fractions (2) with the widely accepted lower limit for the sampling rate of $1 \mathrm{kHz}$ [12], such that for the ranges results $0 \leq \gamma<0.001$ and $0 \leq \delta<0.02$.

These boundaries reveal that the effect of physical damping $\delta$ and stiffness $\gamma$ on stability is rather small. Nevertheless, the presence of these parameters slightly increases the stable region. Moreover, the mass $m$ is linearly scaling the normalized axes and thus the boundaries. Therefore, in the end all three parameters of the human arm contribute to stability.

\section{OPTIMAL RENDERING}

The previous section presented stability boundaries for the investigated haptic system. These boundaries show for which parameters the system is stable, and thus define the maximal stiffness range, resulting as $\alpha \in\left[-\gamma, \alpha_{\max }\right]$ (see Tables III and IV for the numerical values for $d=0$ and $d=1$ ). Although a wide stiffness range is desirable in many cases, it says nothing about the system behavior itself. Thus, the important question of how to set the parameters of a haptic system in an optimal way remains unanswered.

A common way of optimizing system behavior is placing the system poles inside certain regions in the plane of the complex variable [13]. More interesting than pole-based criteria for the practical use are criteria that consider the system response of haptic systems directly. Such response does not only depend on the system poles, but rather on the whole transfer function.

This section introduces several optimization criteria that take the step response of the haptic system into account. Applying a unit step input is equivalent to shifting the equilibrium position of the mass $m$ by $\triangle x=1 /(k+K)$. Based on the step response, this section further determines optimal points inside the stable region. As they are given in normalized parameters, they can be transformed into
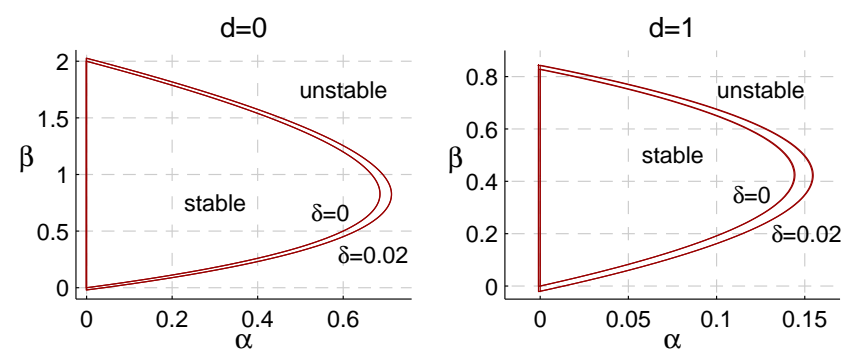

Fig. 3. Stability boundaries in the $(\alpha, \beta)$-plane for $d=0$ (left) and $d=1$ (right), and for the limits of the parameter range $\gamma \in[0,0.001]$ and $\delta \in[0,0.02]$. The effect of $\gamma$ on the stability boundary is not visible in this scale.

their non-normalized counterparts by the normalization rules specified in Table I, i.e.

$$
\begin{aligned}
& K_{\mathrm{opt}}=\alpha_{\mathrm{opt}} \cdot m / T^{2} \\
& B_{\mathrm{opt}}=\beta_{\mathrm{opt}} \cdot m / T .
\end{aligned}
$$

Note that this relation implies a linear dependency on the total mass $m$, and a linear respectively quadratic one on the sampling rate $1 / T$. The first subsection introduces energybased criteria, whereas the second subsection analyzes the overshoot of the haptic system.

\section{A. Fast Energy Dissipation}

One important control design objective for haptic systems is optimizing the settling behavior. To this end, the following lines introduce a criterion that considers how fast energy of the step response is declining.

In general, the considered system can store energy only by the moving mass $m$ and the two springs $k$ and $K$. Under the assumption that the potential energy of the discrete-time spring $K$ has the same equation as an ideal continuous-time spring, the total system energy is constituted by the sum of potential and kinetic energy,

$$
E(t)=\frac{1}{2}(K+k)(x(t)-x(\infty))^{2}+\frac{1}{2} m \dot{x}^{2}(t) .
$$

The continuous-time step response of the investigated haptic system describes the movements $x(t)$ of the mass $m$ caused by a unit step of the input force $F(t)=1 N$ for $t \geq 0$. This input step is introducing energy $E(0)$ into the system and pushing the final position to $x(\infty)=x(0)+F /(k+K)$. As the mass $m$ is not moving before the unit step acts, the initial energy $E(0)$ is given only by the energy stored in the two springs, i.e.

$$
E(0)=\frac{1}{2}(k+K)(x(0)-x(\infty))^{2}=\frac{1}{2} \frac{F^{2}}{k+K} .
$$

To achieve fast settling behavior, this energy should be dissipated as fast as possible. This design goal can be formulated as linear quadratic (LQ) optimal control problem with a cost function given by the time-integral of the system energy. In order to obtain cost values that are independent of the two system parameters $m$ and $T$, this integral is 

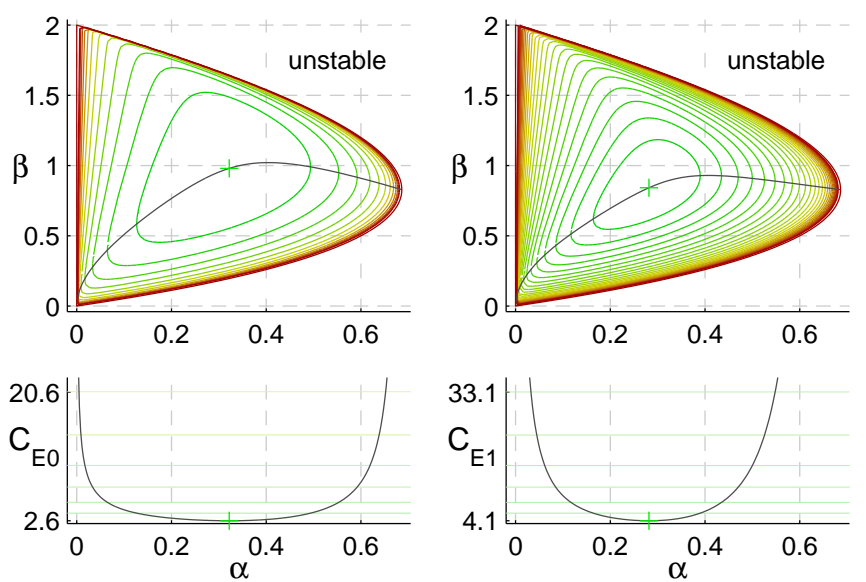

(a) $C_{E 0}$

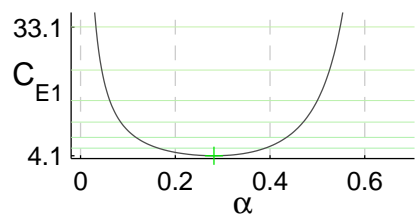

(b) $C_{E 1}$
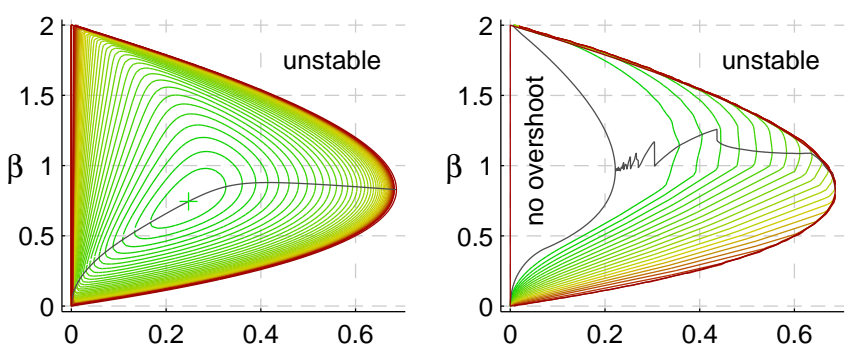

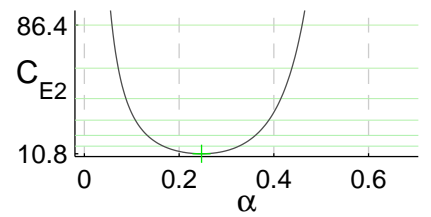

(c) $C_{E 2}$

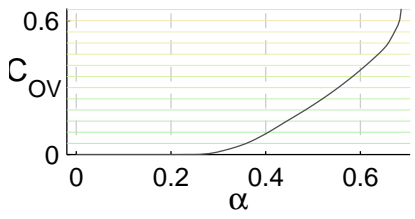

(d) $C_{\mathrm{OV}}$

Fig. 4. The optimal curves for the four analyzed criteria for $d=0, \gamma=0$ and $\delta=0$. Contour lines in the upper subfigures illustrate how the costs are changing. The lower subfigures show the development of costs when moving along the optimal curve.

normalized by the initial energy $E(0)$ and the sampling period $T$, resulting in

$$
C_{E 0}:=\frac{1}{T \cdot E(0)} \int E(t) d t
$$

Motivated by time scaling of standard optimization criteria [11], two more criteria are introduced with cost functions,

$$
\begin{aligned}
C_{E 1} & :=\frac{1}{T^{2} \cdot E(0)} \int E(t) t d t \\
C_{E 2} & :=\frac{1}{T^{3} \cdot E(0)} \int E(t) t^{2} d t
\end{aligned}
$$

where the time $t$ is starting with the input step. They result in optima with faster energy dissipation than $C_{E 0}$, since late energy is penalized more severely. In comparison, criteria that only consider the square error of the position (e.g. [11], [13]), i.e. only the potential energy stored in the springs, result in optimal curves with faster movements and slower dissipation of the total energy than the suggested criteria. On the other hand, if only kinetic energy is considered, optima with extremely slow movements result.

The optimal curves inside the stable parameter region in the $(\alpha, \beta)$-plane can be determined by a method composed of two calculation steps. First, the stable region is gridded and for each grid point the costs $C_{E x}$ are determined. Second, for each grid value of $\alpha$ the optimal $\beta$ is refined iteratively until a predefined accuracy is reached. The resulting optimal curves for the three cost functions $C_{E x}$ are shown in Fig. 4(a)-(c) for the case $d=0, \gamma=0$ and $\delta=0$.

The upper subfigures show the optimal curves inside the normalized $(\alpha, \beta)$-plane as black lines, whereas the lower subfigures show the progress of the cost values along these optimal curves. This progress is also illustrated by contour lines, where every second line means a doubling of costs. It appears that for each criterion there is an absolute minimum for the costs, which is marked by a green plus. Their numerical values are listed in Tables III and IV for $d=0$ and $d=1$ respectively. These tables also show that, similar
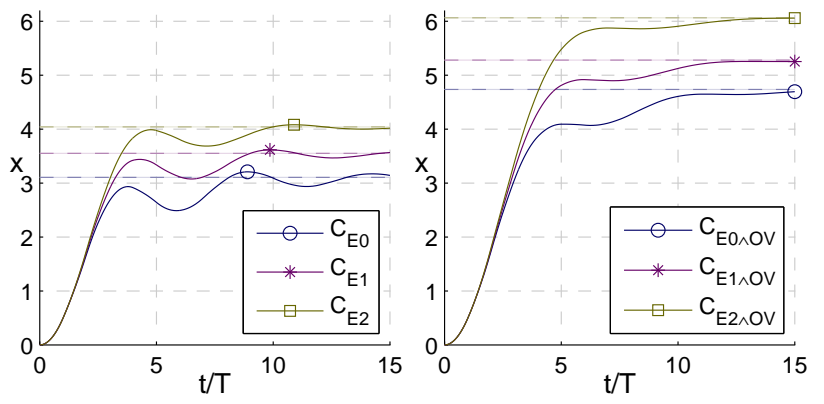

Fig. 5. Continuous-time step responses for optimum points for $d=0$, $\gamma=0$ and $\delta=0$. The markers are placed at the points of maximum overshoot, in case it occurs inside the shown timescale. Left: Optima of the three energy-based criteria $C_{E x}$. Right: Optima of the three energy-based criteria without overshoot $C_{E x \wedge \mathrm{OV}}$.

to the stability boundaries, the influence of $\gamma$ and $\delta$ on the optima is rather small.

The step responses of the haptic system are shown for each of the three optima on the left plot of Fig. 5 exemplarily for $d=0$. For $C_{E 2}$ fastest settling and lowest overshoot are achieved, but with the drawback of foregoing approximately $25 \%$ of virtual stiffness compared to $C_{E 0}$ Therefore, if fast settling is of higher importance than maximizing virtual stiffness, then the most favorable of the three criteria for control design is the optimum point of $C_{E 2}$.

Note that for the three cost functions $C_{E x}$ the continuoustime position signal $x(t)$ was taken into account. The discrete-time position would result in wrong optima with a more than $3 \%$ too high virtual stiffness $\alpha$ for $d=0$. Yet, for higher delays this error becomes much smaller due to the slower dynamics of the haptic system in the optimal points, such that for $d=1$ the error is already less than $1 \%$.

\section{B. Minimum Overshoot}

Another fundamental control design objective is minimizing the overshoot. The overshoot is constituted by the position difference between the steady-state and the maximum position of the step response. The cost function is defined as 


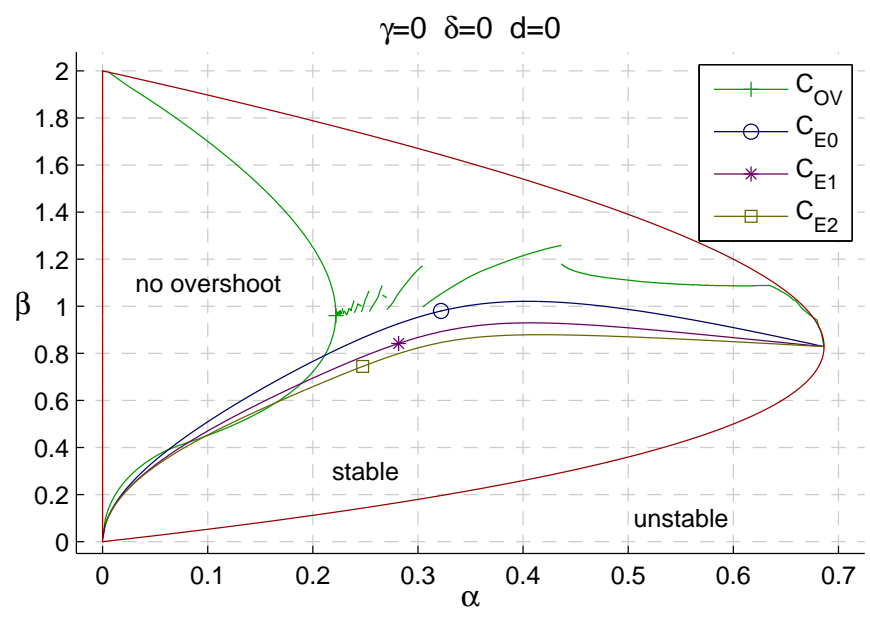

Fig. 6. Optimal curves for delay $d=0$.

the relative overshoot

$$
C_{\mathrm{OV}}:=(\max (x(t))-x(\infty)) / x(\infty) .
$$

For this overshoot criterion an optimal curve can be determined as for the energy-based criteria (see Fig. 4(d)). This curve reveals that in a subregion of the stable region the step response has no overshoot $C_{\mathrm{OV}}=0$. Outside of that subregion the resulting optimal curve is discontinuous. The numerical values of the point with no overshoot and maximum virtual stiffness $\alpha$ (i.e. the rightmost point of the subregion with no overshoot) are listed in Tables III and IV, labeled with $C_{\mathrm{OV}}$.

Yet, for practical applications of higher relevance are optimal points, which have as primary criterion minimum overshoot, and as side criterion fast energy dissipation. Such optima would result in faster settling behavior, while not causing any overshoot. To this end, Fig. 6 shows the three optimal curves for fast energy dissipation in the same plot as the region without overshoot. The optimal points being sought are the rightmost points on the optimal curves, which just do not cause an overshoot (i.e. the right intersection points of the green curve with the three optimal curves).

Their values are also listed in Tables III and IV denoted as $C_{E x \wedge \mathrm{OV}}$. The step responses of these optima are shown in the right plot of Fig. 5 exemplarily for $d=0$. Note that in order to determine the overshoot $C_{\mathrm{OV}}$ of the position of the physical mass $m$ correctly, the continuous-time position $x(t)$ must be taken into account. Only if the maximum position falls just on a sampling instance, it can be determined by the discrete-time signal. Otherwise the real overshoot would always be higher than the one detected.

\section{EXPERIMENTS}

This section describes experiments performed on two haptic devices, the Novint Falcon and the DLR/KUKA LightWeight Robot (LWR). Two different kinds of experiments were performed on each device. The first was used to determine stability boundaries of the devices, while the second resulted optimal curves inside the stable regions.

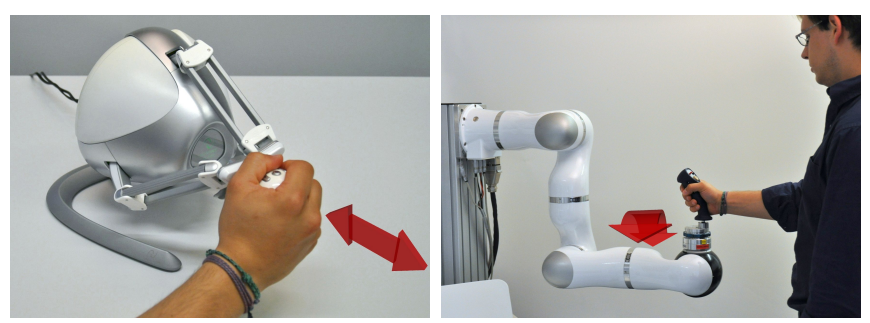

Fig. 7. The configuration of the Falcon (left) and the LWR (right) when performing the experiments. The direction of the movements are indicated by the red arrows.

TABLE II

TOTAL MOVING MASS RESPECTIVELY INERTIA IN THE EXPERIMENTS.

\begin{tabular}{lcc}
\hline & no human & with human \\
\hline Falcon & $0.22 \mathrm{~kg}$ & $0.3 \mathrm{~kg}$ \\
LWR & $0.062 \mathrm{~kg} \mathrm{~m}^{2}$ & $0.065 \mathrm{~kg} \mathrm{~m}^{2}$ \\
\hline
\end{tabular}

These optimal curves followed the optimization criteria (6)(9). Both kinds of experiments were performed with and without a human holding the devices. The human operator was told to hold each devices in a comfortable manner with medium force. The determined inertia $m$ of each experiment is shown in Table II.

For the first kind of experiments a bidirectional wall consisting of a discrete-time spring $K$ and damper $B$ was implemented in one degree of freedom, as indicated in Fig. 7. For each value in a predefined set of reasonable $B$, a limit stable virtual stiffness $K$ was determined by a two steps method, similar to the one used for the theoretical curves in the previous section. For the second set of experiments, the data acquired in the previous kind of experiments was used to predefine stable parameter ranges. Inside these ranges the same gridding method was used, but this time to determine optimal $B$ with minimum costs $C_{E x}$ and $C_{\mathrm{OV}}$.

\section{A. Falcon}

The Falcon is a low-cost commercial haptic device with parallel kinematics (Fig. 7, left). The handle is linked to the base through three symmetrically arranged arms. The device was connected via USB to a standard Linux computer without a real-time operating system. The execution speed of the controller program was paused each sampling step to obtain an average sampling rate of $1 \mathrm{kHz}$. The overall average delay was $6 \mathrm{~ms}$.

Fig. 8(a) shows the stability boundaries and the optimal curves and points. Due to noise, mostly present at high virtual stiffness and damping there are small peaks in both, stability boundaries and cost functions. The experiments performed with a human operator show a considerable increase of the stable region towards higher $K$ and $B$, and similarly a scale of the optimal curves. Following the theoretical analysis this increase originates mainly from the additional mass introduced by the human operator [9]. The human also shifts the starting point of the stability boundary at $B=0$ towards around $K \approx 6.000 \mathrm{~N} / \mathrm{m}$. This shift can be explained by the additional physical damping introduced by the human arm. 

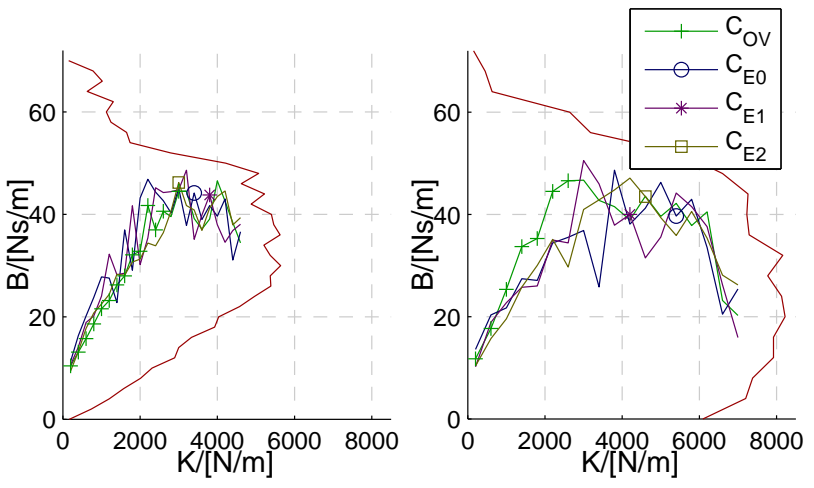

(a) Results of the experiments performed on the Falcon
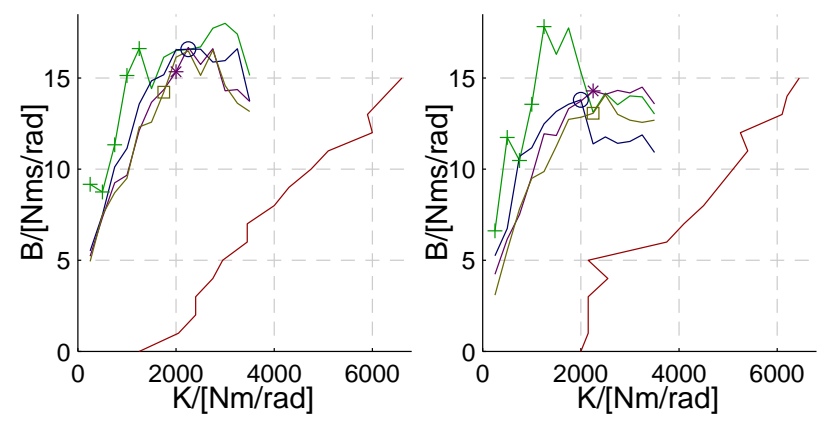

(b) Results of the experiments performed on the LWR

Fig. 8. The experimental stability boundaries and optimal curves for the two sets of experiments (left: no human; right: with human) for the Falcon device (top) and LWR (bottom). The pluses indicate zero overshoot. The other three markers indicate the optimal points for the criteria $C_{E x}$.

\section{B. Light-Weight Robot}

The LWR is a seven degree of freedom robot arm equipped with torque sensors in every joint [14], [15]. Though it weighs only $14 \mathrm{~kg}$, it is able to handle payloads of $7 \mathrm{~kg}$ throughout the whole dynamic range. The experiments were performed on the fifth robot joint (see Fig. 7, right). The environment was implemented using a real-time computer connected to the robot via sercos interface. The sampling rate was constant at $1 \mathrm{kHz}$ and a round trip delay of $5 \mathrm{~ms}$ was determined, which has its origin in the specific hardware and software infrastructure used.

The curved shape of the theoretical stability boundaries could not be determined by this experimental configuration (see Fig. 8(b)). As the robot should not be stressed too much by the stability tests, the experiments were stopped at a virtual damping of $15 \mathrm{Nm} \mathrm{s} / \mathrm{rad}$. A possible reason why the curved shape did not occur, may be due to elasticity in the mounting of the LWR. Nevertheless, the optimal curves were determined. Compared to the Falcon device, the human effect is not as strong in the experimental results. This is probably because the human has a relatively lower contribution to the total moving inertia $m$ in this experiment (see Table II).

\section{CONCLusions}

The present article suggests optimization criteria based on the step response of haptic systems and determines optimal curves and points for each of these criteria. They cover two fundamental requirements for haptic systems, i.e. fast settling and minimum overshoot. Similar to the stability boundaries, the influence of the human on the determined optimal curves and points is mainly constituted by the arm mass, since they depend linearly on the total system mass. In comparison, these optima are nearly not affected by the human stiffness and damping using realistic parameter ranges.

One important outcome of the performed analyses is that the stable regions are not flat with respect to the considered cost functions. Rather, there is an optimal virtual damping for each virtual stiffness, depending on the optimization criterion chosen. It is remarkable that a further increase of the virtual damping above the optimal curves does not speed up energy dissipation with regard to the step response. A main reason therefore may be that a higher damping factor slows down movements and causes the system to take longer to reach its steady-state position.

It is interesting to note that there is also a certain optimal virtual stiffness, with its corresponding damping value. Above that optimal stiffness value the system behaves worse with respect to the optimization criteria. Therefore, the virtual wall parameters cannot be chosen arbitrarily inside the stable region, without highly affecting system behavior. Rather, they have to be carefully tuned, by weighting up the system behavior and the desired stiffness that is demanded for example by a virtual reality simulation. This finding contrasts classical approaches that aim at purely maximizing stable virtual stiffness, e.g. [2], [16].

The step response, which constitutes the basis for the optimality analysis presented in this article, corresponds to the response of the haptic system to a shift of the equilibrium position of the mass. Thus, on the other hand, the specific optimal curves and points presented in this article do not hold for haptic devices colliding with a certain initial velocity against a virtual wall. To find optimal solutions for that case, the impulse response of the analyzed haptic system must be considered. The difference between the two is that the impulse response corresponds to the case with initial kinetic energy, whereas the step response implies initial potential energy. Preliminary investigations indicate that the optimal curves for the impulse response look quite different to those derived in the present article. But nevertheless, above general conclusions and relations still hold for the impulse response.

Finally, it is revealing to apply the passivity condition [1] to these optimal points. It can be easily checked that they violate passivity (see also [9]). This finding does not only emphasizes the fact that passivity is conservative for stable control of haptic systems, but rather suggests a detrimental property that passivity prevents haptic systems of being controlled in an optimal way.

The devices on which the experiments were performed were affected by quite long time delay of several sampling steps. It would be interesting for the future work to analyze the optimization criteria also on haptic devices with very short delays. Possibly, nonlinear effects, which were neglected in the present approach, will have a predominant effect on stability. 
TABLE III

OPTIMAL VALUES FOR THE CRITERIA FOR DELAY $d=0$.

\begin{tabular}{cccccc}
\hline criterion & \multicolumn{2}{c}{ given parameters } & \multicolumn{3}{c}{ resulting optimum } \\
& $\gamma$ & $\delta$ & $\alpha_{\text {opt }}$ & $\beta_{\text {opt }}$ & cost \\
\hline \hline \multirow{6}{*}{$\alpha_{\max }$} & 0.000 & 0.00 & 0.6863 & 0.8283 & - \\
& 0.001 & 0.00 & 0.6858 & 0.8280 & - \\
& 0.000 & 0.02 & 0.7132 & 0.8244 & - \\
& 0.001 & 0.02 & 0.7127 & 0.8241 & - \\
\hline \multirow{6}{*}{$C_{\mathrm{OV}}$} & 0.000 & 0.00 & 0.222 & 0.97 & 0.000 \\
& 0.001 & 0.00 & 0.221 & 0.97 & 0.000 \\
& 0.000 & 0.02 & 0.230 & 0.97 & 0.000 \\
& 0.001 & 0.02 & 0.230 & 0.97 & 0.000 \\
\hline \multirow{6}{*}{$C_{E 0}$} & 0.000 & 0.00 & 0.3220 & 0.9800 & 2.583 \\
& 0.001 & 0.00 & 0.3212 & 0.9798 & 2.582 \\
& 0.000 & 0.02 & 0.3341 & 0.9816 & 2.536 \\
& 0.001 & 0.02 & 0.3333 & 0.9815 & 2.535 \\
\hline \multirow{6}{*}{$C_{E 1}$} & 0.000 & 0.00 & 0.2816 & 0.8419 & 4.143 \\
& 0.001 & 0.00 & 0.2808 & 0.8417 & 4.140 \\
& 0.000 & 0.02 & 0.2922 & 0.8415 & 4.002 \\
& 0.001 & 0.02 & 0.2914 & 0.8413 & 3.999 \\
\hline \multirow{6}{*}{$C_{E 2}$} & 0.000 & 0.00 & 0.2474 & 0.7445 & 10.80 \\
& 0.001 & 0.00 & 0.2466 & 0.7443 & 10.79 \\
& 0.000 & 0.02 & 0.2569 & 0.7427 & 10.26 \\
& 0.001 & 0.02 & 0.2561 & 0.7425 & 10.25 \\
\hline \multirow{6}{*}{$C_{E 0 \wedge \mathrm{OV}}$} & 0.000 & 0.00 & 0.211 & 0.791 & 2.877 \\
& 0.001 & 0.00 & 0.210 & 0.791 & 2.876 \\
& 0.000 & 0.02 & 0.219 & 0.788 & 2.827 \\
& 0.001 & 0.02 & 0.218 & 0.788 & 2.826 \\
\hline \multirow{6}{*}{$C_{E 1 \wedge \mathrm{OV}}$} & 0.000 & 0.00 & 0.189 & 0.673 & 5.136 \\
& 0.001 & 0.00 & 0.189 & 0.673 & 5.132 \\
& 0.000 & 0.02 & 0.197 & 0.670 & 4.962 \\
& 0.001 & 0.02 & 0.196 & 0.670 & 4.959 \\
\hline & 0.000 & 0.00 & 0.165 & 0.591 & 15.51 \\
& 0.001 & 0.00 & 0.164 & 0.590 & 15.49 \\
& 0.000 & 0.02 & 0.171 & 0.586 & 14.77 \\
& 0.001 & 0.02 & 0.170 & 0.586 & 14.75 \\
\hline
\end{tabular}

\section{ACKNOWLEDGMENT}

The authors would like to acknowledge the fruitful discussions with Moritz Maier, Martin Arbesmeier and Philipp Kremer. The experiments described in Sect. V were performed within the scope of an internship of Ricardo González Camarero under the supervision of the other two authors.

\section{REFERENCES}

[1] J. E. Colgate and G. Schenkel, "Passivity of a class of sampled-data systems: Application to haptic interfaces," Journal of Robotic Systems, vol. 14, no. 1, pp. 37-47, Jan. 1997.

[2] T. Hulin, C. Preusche, and G. Hirzinger, "Stability boundary for haptic rendering: Influence of physical damping," in Proc. IEEE/RSJ Int. Conf. on Intelligent Robots and Systems (IROS), Beijing, China, Oct. 2006, pp. 1570-1575.

[3] B. Hannaford and J.-H. Ryu, "Time-domain passivity control of haptic interfaces," IEEE Trans. on Robotics and Automation, vol. 18, no. 1, pp. 1-10, 2002.

[4] J.-H. Ryu, C. Preusche, B. Hannaford, and G. Hirzinger, "Time domain passivity control with reference energy behavior," IEEE Trans. on Control Systems Technology, vol. 13, no. 5, pp. 737-742, Sep. 2005.

[5] K. Hertkorn, T. Hulin, P. Kremer, C. Preusche, and G. Hirzinger, "Time domain passivity control for multi-degree of freedom haptic systems with time delay," in Proc. IEEE Int. Conf. on Robotics and Automation (ICRA), Anchorage, Alaska, USA, May 2010.

[6] C. Ott, J. Artigas, and C. Preusche, "Subspace-oriented energy distribution for the time domain passivity approach," in Proc. IEEE/RSJ Int. Conf. on Intelligent Robots and Systems (IROS), San Francisco, CA, USA, Sep. 2011, pp. 665-671.
TABLE IV

OPTIMAL VALUES FOR THE CRITERIA FOR DELAY $d=1$.

\begin{tabular}{cccccc}
\hline criterion & \multicolumn{2}{c}{ given parameters } & \multicolumn{3}{c}{ resulting optimum } \\
& $\gamma$ & $\delta$ & $\alpha_{\text {opt }}$ & $\beta_{\text {opt }}$ & cost \\
\hline \hline \multirow{6}{*}{$\alpha_{\max }$} & 0.000 & 0.00 & 0.1445 & 0.4247 & - \\
& 0.001 & 0.00 & 0.1440 & 0.4238 & - \\
& 0.000 & 0.02 & 0.1548 & 0.4227 & - \\
& 0.001 & 0.02 & 0.1543 & 0.4220 & - \\
\hline \multirow{4}{*}{$C_{\text {OV }}$} & 0.000 & 0.00 & 0.052 & 0.45 & 0.000 \\
& 0.001 & 0.00 & 0.051 & 0.45 & 0.000 \\
& 0.000 & 0.02 & 0.056 & 0.45 & 0.000 \\
& 0.001 & 0.02 & 0.055 & 0.45 & 0.000 \\
\hline \multirow{6}{*}{$C_{E 0}$} & 0.000 & 0.00 & 0.0702 & 0.4710 & 5.429 \\
& 0.001 & 0.00 & 0.0695 & 0.4705 & 5.419 \\
& 0.000 & 0.02 & 0.0753 & 0.4718 & 5.249 \\
& 0.001 & 0.02 & 0.0745 & 0.4713 & 5.240 \\
\hline \multirow{6}{*}{$C_{E 1}$} & 0.000 & 0.00 & 0.0642 & 0.4191 & 17.35 \\
& 0.001 & 0.00 & 0.0634 & 0.4185 & 17.29 \\
& 0.000 & 0.02 & 0.0688 & 0.4188 & 16.26 \\
& 0.001 & 0.02 & 0.0680 & 0.4182 & 16.21 \\
\hline \multirow{6}{*}{$C_{E 2}$} & 0.000 & 0.00 & 0.0582 & 0.3796 & 87.44 \\
& 0.001 & 0.00 & 0.0574 & 0.3790 & 86.97 \\
& 0.000 & 0.02 & 0.0625 & 0.3786 & 79.40 \\
& 0.001 & 0.02 & 0.0618 & 0.3778 & 79.06 \\
\hline \multirow{6}{*}{$C_{E 0 \wedge \mathrm{OV}}$} & 0.000 & 0.00 & 0.051 & 0.407 & 5.832 \\
& 0.001 & 0.00 & 0.050 & 0.407 & 5.821 \\
& 0.000 & 0.02 & 0.055 & 0.406 & 5.641 \\
$C_{E 2 \wedge \mathrm{OV}}$ & 0.001 & 0.02 & 0.054 & 0.405 & 5.631 \\
\hline \multirow{6}{*}{$C_{E 1 \wedge \mathrm{OV}}$} & 0.000 & 0.00 & 0.048 & 0.356 & 20.16 \\
& 0.001 & 0.00 & 0.047 & 0.356 & 20.09 \\
& 0.000 & 0.02 & 0.051 & 0.354 & 18.90 \\
& 0.001 & 0.02 & 0.050 & 0.353 & 18.84 \\
\hline & 0.000 & 0.00 & 0.043 & 0.318 & 113.8 \\
& 0.000 & 0.00 & 0.042 & 0.317 & 113.2 \\
& 0.001 & 0.02 & 0.046 & 0.315 & 102.5 \\
& & & 0.045 & 0.314 & 102.8 \\
\hline
\end{tabular}

[7] S. E. Salcudean and T. D. Vlaar, "On the emulation of stiff walls and static friction with a magnetically levitated input/output device," ASME Journal of Dynamic Systems, Measurement and Control, vol. 119, no. 1, pp. 127-132, Mar. 1997.

[8] J. J. Gil, E. Sánchez, T. Hulin, C. Preusche, and G. Hirzinger, "Stability boundary for haptic rendering: Influence of damping and delay," in Proc. IEEE Int. Conf. on Robotics and Automation (ICRA), Rome, Italy, Apr. 2007.

[9] T. Hulin, C. Preusche, and G. Hirzinger, "Stability boundary for haptic rendering: Influence of human operator," in Proc. IEEE/RSJ Int. Conf. on Intelligent Robots and Systems (IROS), Nice, France, Sep. 2008, pp. 3483-3488.

[10] J. J. Gil, A. Avello, A. Rubio, and J. Flórez, "Stability analysis of a 1 dof haptic interface using the Routh-Hurwitz criterion," IEEE Trans. on Control Systems Technology, vol. 12, no. 4, pp. 583-588, Jul. 2004.

[11] S. M. Shinners, Modern Control System Theory and Design, 2nd ed. John Wiley and Sons, Inc., 1998, ch. Performance Criteria.

[12] C. Basdogan and M. A. Srinivasan, "Haptic rendering in virtual environments," in Handbook of Virtual Environments: Design, Implementation, and Applications, K. Stanney, Ed. Erlbaum, 2002, pp. $117-134$.

[13] T. Hulin, C. Preusche, and G. Hirzinger, "Stability boundary and design criteria for haptic rendering of virtual walls," in Proc. Int. IFAC Symp. on Robot Control (SYROCO), Bologna, Italy, Sep. 2006.

[14] G. Hirzinger, N. Sporer, M. Schedl, J. Butterfaß, and M. Grebenstein, "Torque-controlled lightweight arms and articulated hands: Do we reach technological limits now?" The International Journal of Robotics Research, vol. 23, no. 4-5, pp. 331-340, 2004.

[15] T. Hulin, K. Hertkorn, P. Kremer, S. Schätzle, J. Artigas, M. Sagardia, F. Zacharias, and C. Preusche, "The DLR bimanual haptic device with optimized workspace," in Proc. IEEE Int. Conf. on Robotics and Automation (ICRA), Shanghai, China, May 2011, pp. 3441-3442.

[16] J. E. Colgate and J. M. Brown, "Factors affecting the z-width of a haptic display," in Proc. IEEE Int. Conf. on Robotics and Automation (ICRA), May 1994, pp. 3205-3210. 